\title{
An effective microscale approach for determining the aniostropy of polymer composites reinforced with randomly distributed short fibers
}

\author{
Heng Cai ${ }^{\mathrm{a}}$, Junjie Ye $\mathrm{e}^{\mathrm{a}, \mathrm{b}^{*}}$, Yiwei Wang ${ }^{\mathrm{a}}$, Mohammed Saafi ${ }^{\mathrm{b}}$,
} Bo Huang ${ }^{\mathrm{b}}$, Dongmin Yang ${ }^{\mathrm{c}}$, Jianqiao Ye $^{\mathrm{b} *}$

\footnotetext{
${ }^{a}$ Research Center for Applied Mechanics, Key Laboratory of Ministry of Education for Electronic Equipment Structure Design, Xidian University, Xi’an 710071, China

${ }^{\mathrm{b}}$ Department of Engineering, Lancaster University, Lancaster LA1 4YW, UK

${ }^{c}$ Institute for Materials and Processes, school of engineering, university of Edinburgh, EH9 3FB, Edinburgh, UK
}

\begin{abstract}
In this paper, an effective microscopic modeling scheme is presented to analyze mechanical properties of composites with random short fibers. To this end, the displacement-load tests of the standard samples, which are acquired by cutting a short fiber-reinforced composite plate of $650 \mathrm{~mm} \times 650 \mathrm{~mm} \times 2.5 \mathrm{~mm}$, are firstly executed under the quasi-static tensile loads. To identify the geometric sizes of the short fibers and their distributions at microscopic scale, the advanced microcomputed tomography (micro-CT) is employed by testing a small sample of $1 \mathrm{~cm} \times 2.5 \mathrm{~mm} \times 2.5 \mathrm{~mm}$. On this basis, a simplified microscopic model is reconstructed by the 3D parametric finite-volume direct averaging micromechanics (FVDAM) theory according to the statistic results of the microCT images. The proposed method is further validated by comparing the effective modulus obtained from tensile tests. The scanning electron microscopy (SEM) is also used to visualize the fracture morphology of the fibers. It is found that brittle fracture occurs in the short-fibers paralleled to the external loading.
\end{abstract}

Keywords: Random short fibers, SFRCs, Fracture morphology, Micro-CT.

\section{Introduction}

Composites, one of the most popular engineering materials, have been widely used in various industry due to their excellent performances [1-4]. Compared with continuous fiber-reinforced composites, short fiber-reinforced composites (SFRCs) always exhibit a better fracture toughness [5-6]. In recent years, new additive manufacturing technologies [8-10] provide an important opportunity for the SFRCs, and significantly reduce internal voids during the forming process. Naturally, their mechanical properties, such as tensile and flexural strengths, will be greatly improved. However, understanding their failure mechanisms is a challenge topic for researchers due to their complex and unpredictable microstructure.

Advanced experimental techniques, such as scanning electron microscopy (SEM) and microcomputed tomography (micro-CT), are important analytical tools that provide objective basis to appreciate the mechanical properties of composite materials at microscale. Three-dimensional micro-morphology can be reconstructed by SEM through collecting the secondary electrons [11]. Rolland et al. [12] captured the damage processes of glass short-fiber reinforced composites by insitu SEM. Shi et al. [13] evaluated the shear failures of short carbon fibers in ceramic matrix materials by SEM. However, SEM is restricted to discern surface cracks or damages. It is no doubt that micro-CT, which is a technology supplement of SEM, can be employed to discern internal damages in a composite material system [10,14-16]. This is attributed to the composition element differences between reinforced inclusions and matrix materials, which can be easily distinguished by the attenuation of the X-ray. Wu et al. [17] validated the correctness of the flow simulation in injection molding (IM) process of SFRCs based on the micro-CT scanning results. Thi et al. [18]

\footnotetext{
*Corresponding authors.

Email address: ronkey6000@sina.com (Junjie Ye)

j.ye2@lancaster.ac.uk (Jianqiao Ye)
} 
employed micro-CT to obtain fiber orientations and microstructures to determine the effects of micro-morphology on the mechanical properties of SFRCs.

At present, IM and hot/cold compression molding (CM) have been widely used in the forming process of SFRCs [19-20]. Many researchers have devoted themselves to establish a micromechanical model to investigate the mechanical behaviors of composites made by IM technology [21-22]. Dean et al. [23-24] established a three-dimensional thermoplastic coupling model to simulate mechanical properties of the IM-formed SFRCs with finite element method (FEM). Tanaka et al. [25] evaluated the fatigue crack growths of the IM-formed laminates by FEM. Some researchers recommended that a transversal isotropic model could be used to describe SFRCs with spatial random distribution of short fibers made by IM technology, while the SFRCs made by CM technology are obviously anisotropic. Some studies concentrated on establishing simplified anisotropic microscopic models to analyze effective properties of composites with randomly distributed fibers in the matrix materials [26]. Qin et al. [27] presented a simplified micromechanical model of dental composites with a cylindrical structure to determine the effective mechanical properties, which is not readily suitable for composites with randomly distributed short fibers. In general, real microscopic morphology and fiber arrangements in the matrix material are always ignored in establishing the microscopic model to evaluate the anisotropic mechanical properties of SFRCs.

In this paper, the random distributions of short fibers at microscopic scale are tested by micro-CT technology. According to the statistical results of fiber length, fiber spatial orientation, a simplified ellipsoid model is proposed to effectively study the anisotropic behaviors of the SFRCs. Elastic moduli obtained by the simplified numerical model are compared with experimental results under the quasi-static tensions. A brief outline of the paper is as follows: The experimental process of the SFCRs under quasi-static tension loadings is introduced in Section 2. Model reconstruction and numerical simulation of the SFRCs based on statistical results by micro-CT technology are presented in Section 3. Descriptions of the fracture surface morphology, as well as revelations of fiber failure behaviors are given in Section 4. The main results are discussed in Section 5.

\section{Quasi-static tensile tests}

\subsection{Experimental preparations}

The tested composite samples, are composed of Polyetheretherketone (PEEK) resins with $30 \%$ mass content of short glass fibers (Ensinger GmbH, Nufringen, Germany), which were processed by compression molding, as shown in Fig. 1. The liquid resin mixed with short fibers flows into the mold as shown in Fig. 1(a). A uniform pressure is applied on the upper surface of the composite plate as shown in Fig. 1(b). The composite plate is then solidified by uniform cooling. Obvious directionality of the short fibers is found due to this preparation technology. The density of PEEKGF30 (Polyetheretherketone, 30\% Short Glass Fiber Reinforced) is $1.53 \mathrm{~g} / \mathrm{cm}^{3}$. Constituent material parameters of the short fibers and the PEEK, which are provided by Owens Corning and Ensinger incorporated, can be found in Table 1. The elastic modulus and tensile strength at room temperature are $6.4 \mathrm{GPa}$ and $105 \mathrm{MPa}$, respectively. It should be noted that the short fibers, whose length is from $20 \mu \mathrm{m}$ to $150 \mu \mathrm{m}$, are obtained by cutting the continuous long fibers. The fiber diameter is approximate to $14 \mu \mathrm{m}$. 
Table 1. Parameters of constituent materials at $23^{\circ} \mathrm{C}$

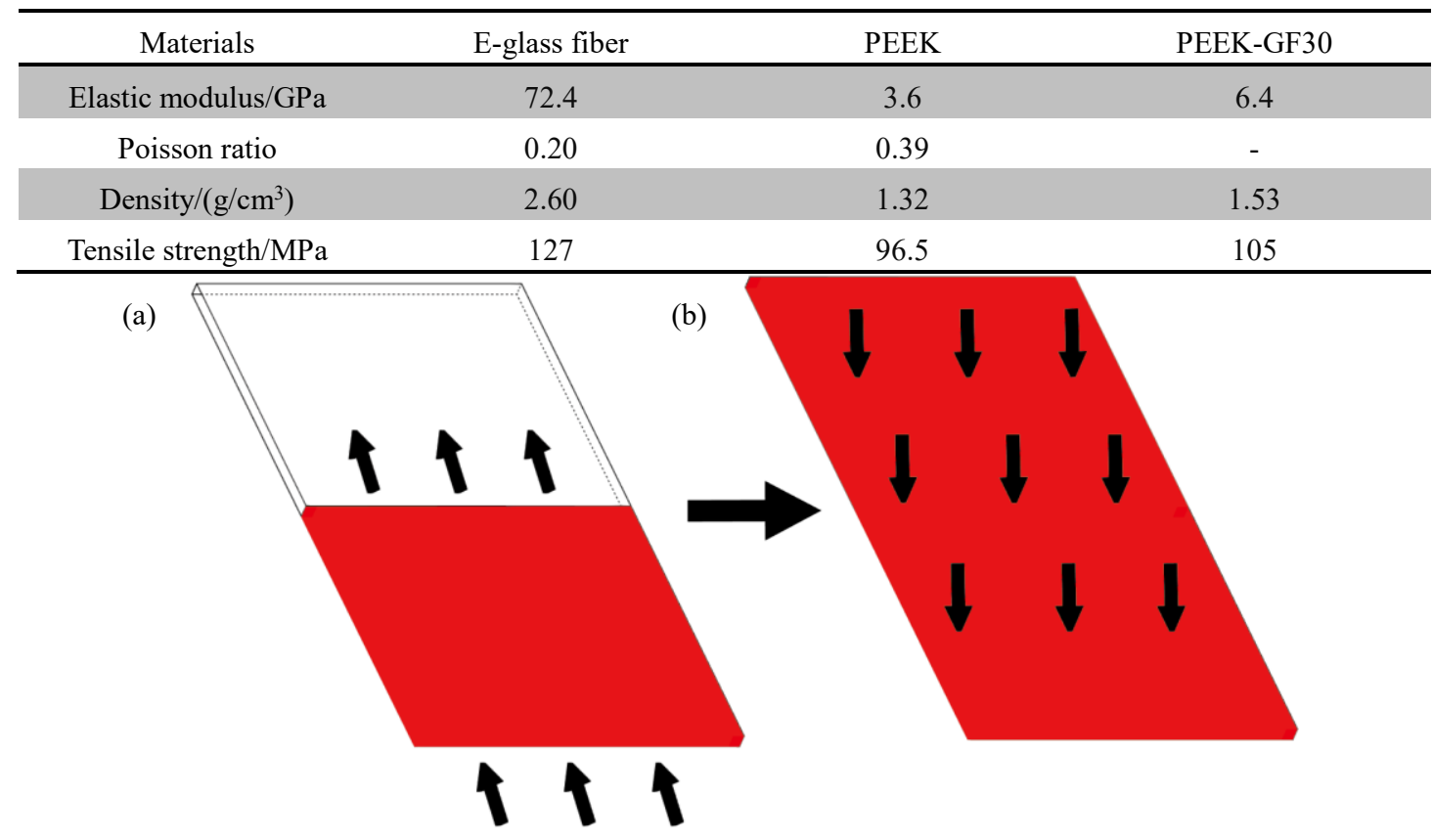

Fig. 1. Schematic diagram of compressive molding: (a) unidirectional flow of resin (b) uniform pressure on the upper surface of the composite plate

According to ASTM D3039/D3039M-17, the dimension of the tensile specimens is $250 \mathrm{~mm} \times 25 \mathrm{~mm} \times 2.5 \mathrm{~mm}$ as shown in Fig. 2(a). The specimens with three off-axis angles $0^{\circ}, 45^{\circ}$ and $90^{\circ}$ (Fig. 2 (b)) are obtained by cutting a composite plate $(650 \mathrm{~mm} \times 650 \mathrm{~mm} \times 2.5 \mathrm{~mm})$, which is made by $\mathrm{CM}$ forming technology. During the cutting process, Computerized Numerical Control (CNC) Series ZMC-L850 is employed to ensure a position precision of $\pm 0.005 / 300 \mathrm{~mm}$. The quasistatic tensile tests are executed by the electronic universal test system DNS100 to acquire stressstrain behaviors of the SFRCs at a constant strain rate $10^{-4} / \mathrm{s}$ (see in Fig. 2(c)).

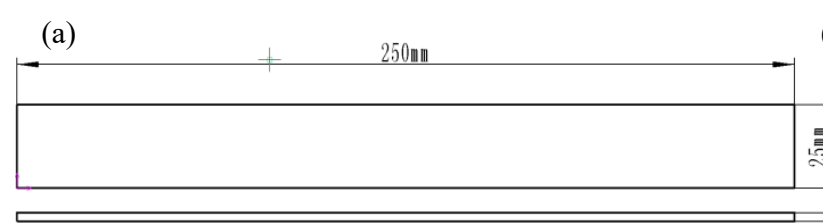

(b)

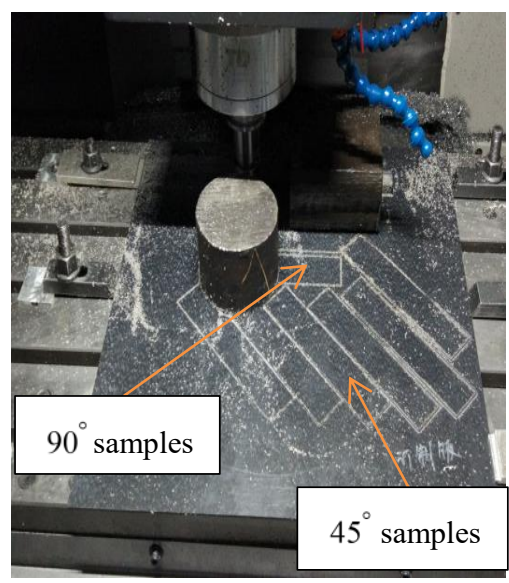

(c)

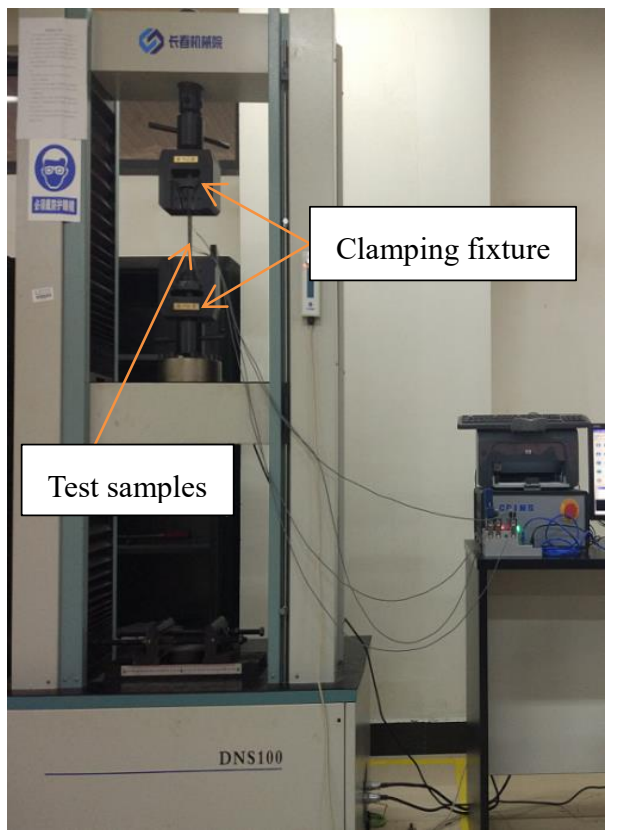

Fig. 2. Parameters of samples and experimental process: (a) Geometric parameters of specimens (b)

Processing the test samples with the CNC (c) The electronic universal test system DNS100 


\subsection{Results of tensile tests}

The displacement-load curves of the SFRCs are obtained under the tensile tests as shown in Fig. 3. Here, the labels of the curves are in the form of PEEK/GL30-W-U, where W and U represent the tailoring direction and serial number of the samples, respectively. From the test results, nonlinear behaviors can be seen after a brief stage of linear elasticity. Moreover, the load capacity closely depends on the cutting angles. In details, the $0^{\circ}$ and $90^{\circ}$ SFRCs provide the highest and lowest load capacity, respectively. In addition, the stiffness of the three $0^{\circ}$ specimens shows noticeable differences, which is attributed to uncertainty of the complex microstructure of the SFRCs.

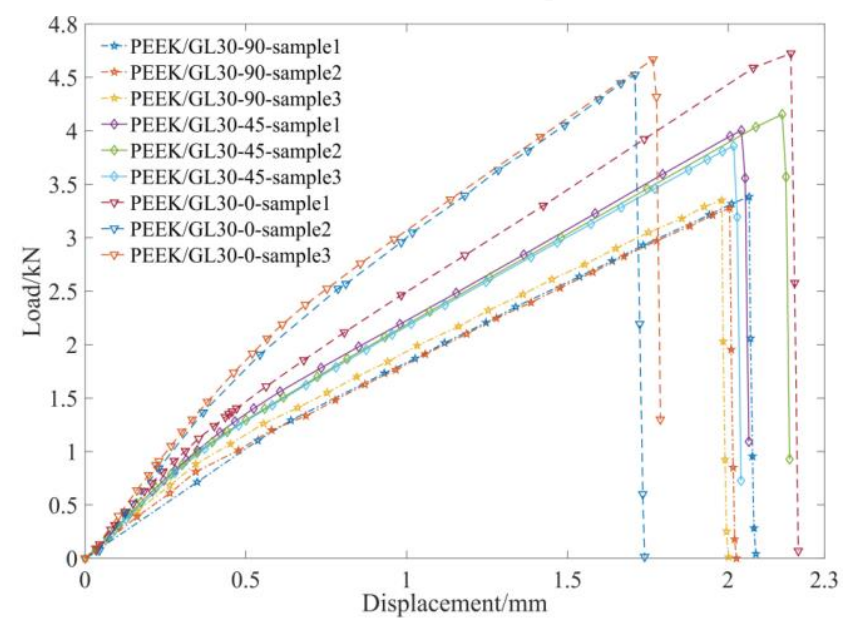

Fig. 3. Displacement-load curves of samples at strain rate of $0.0001 / \mathrm{s}$

To acquire their elastic modulus by the test method, the displacement-load curve shown in Fig. 3 is employed. Note that, the determination of elastic modulus should be lower than the proportional limit in the curves according to the test standard ASTM E111-17. Herein, the tensile elastic modulus of the SFRCs can be calculated by the following equations.

$$
\begin{gathered}
\Delta \varepsilon=\frac{d_{0.05}-d_{0}}{l_{\text {sample }}} \\
E_{\text {sample }}=\frac{f_{0.05}-f_{0}}{S \times \Delta \varepsilon}
\end{gathered}
$$

where $d_{0.05}$ and $l_{0.05}$ represent the maximum tensile displacement and load, respectively. $d_{0}$ and $l_{0}$ represent the displacement and load under an initial loading, respectively. The symbol $S$ represents the cross-section area of each sample. In each test, the dimension of the specimens is accurately measured by vernier caliper. Experimental results of the tensile moduli are shown in Table 2. It can be found that the anisotropic properties of the SFRCs are closely related to the cutting direction. Moreover, the effective modulus shows good consistency across all the samples when the cutting direction is considered.

Table 2. Tested results of tensile modulus of the SFRCs at room temperature

\begin{tabular}{cccc}
\hline Elastic modulus/GPa & $0^{\circ}$ & $45^{\circ}$ & $45^{\circ}$ \\
\hline Sample \#1 & 5.828 & 4.530 & 3.683 \\
Sample \#2 & 6.064 & 4.369 & 3.919 \\
Sample \#3 & 6.159 & 4.521 & 3.943 \\
Average & 6.017 & 4.473 & 3.849 \\
\hline
\end{tabular}

\section{Micro-CT tests and numerical analysis}




\subsection{Microscopic modeling procedures}

To consider microscopic structure and improve calculation efficiency in predicting mechanical properties of composites with random distributions of short fibers, a simplified microscopic model is proposed on the basis of the Micro-CT results. The model is then validated by comparing with the tensile test results in Section 2. The proposed procedure, as shown in Fig. 4, can be divided into the following three main steps: First, advanced micro-CT scanning is employed to accurately obtain the real microscopic morphology and fiber arrangements in the matrix material. Secondly, according to the statistical results of short fiber distributions through the micro-CT, a 3D spherical model is developed. Finally, the effective moduli of the proposed 3D spherical model are calculated and validated by comparing with experimental results.

\subsection{Computed tomography}

To acquire microscopic arrangements of the short fibers in composites, a commercial X-ray micro-CT (Zeiss Xradia Versa 410) is used. The voltage and power of the micro-CT are $80 \mathrm{keV}$ and $7 \mathrm{~W}$, respectively. The flat panel detector provides pixels of $1000 \times 1000$. For each case, 1600 projection images are employed and reconstructed to establish a 3D model by using the standard iterative reconstruction algorithm. The 3D viewer software supplied by the Zeiss Xradia Versa is used to process the data. Qualitative analyses of the images, including fiber orientation and porosity calculation, etc, are carried out on a high-performance workstation using software AVIZO.

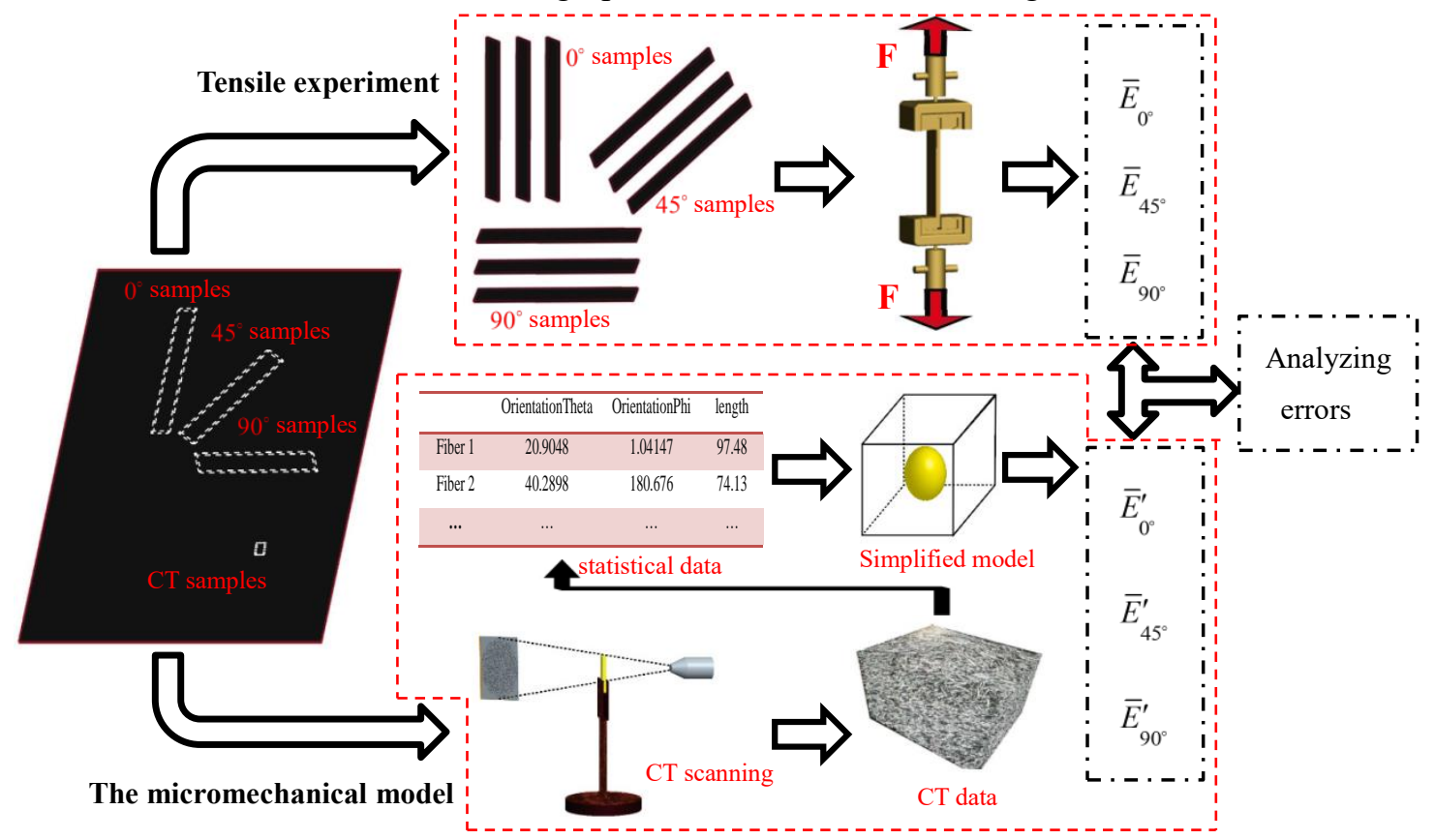

Fig. 4. Schematic diagram of micromechanical model

\subsection{Simplified model}

To acquire the statistical results of the short fiber arrangement, a small sample of $1 \mathrm{~cm} \times 2.5 \mathrm{~mm} \times 2.5 \mathrm{~mm}$ cutting from the tensile specimen is chosen. By employing the image postprocessing software, a cylindrical model of $2 \mathrm{~mm}$ in diameter and $2 \mathrm{~mm}$ in height is established, as shown in Fig. 5(a). To capture the fiber information with a higher accuracy, the image of a $300 \mu \mathrm{m} \times 300 \mu \mathrm{m} \times 300 \mu \mathrm{m}$ sample derived from the cylindrical model is further reconstructed as shown in Fig. 5(b). It should be pointed out that the any bending of the fibres caused by the matix flow during the material forming process is ignored and the fiber length used in this study is determinted 
by its chord length, as shown in Fig. 5(c). The mean length of the fibers is $85.423 \mu \mathrm{m}$.

(a)

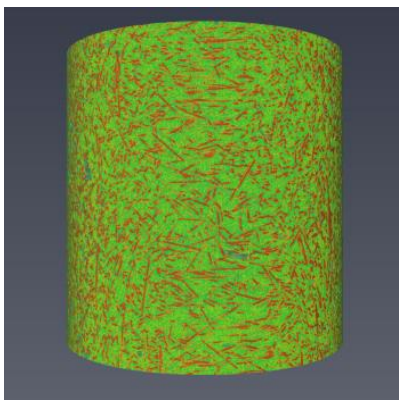

(b)

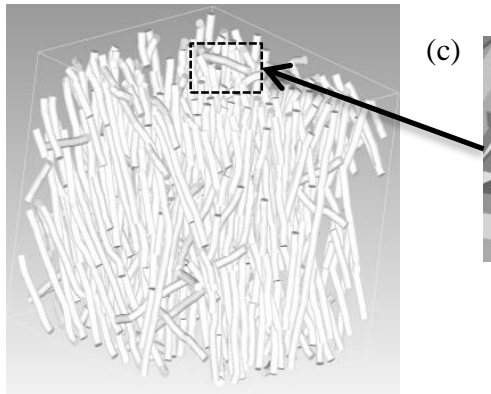

(c)

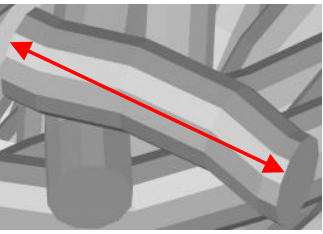

Chord length

Fig. 5. Post-processing data: (a) Micro-topography of the SFRCs through Micro-CT Scanning

(b) $300 \mu \mathrm{m} * 300 \mu \mathrm{m} * 300 \mu \mathrm{m}$ sample reconstruction (c) Partial enlarged graph of the bending fibers

In this section, a simplified three-dimensional representative volume element (RVE) is presented after the statistical analysis of the fiber orientation projections on the $x-y$ and $y-z$ planes. The schematic diagram of the projections on the $x-y$ plane is shown in Fig. 6. As shown in Fig. 6(a), the red line on the $x-y$ plane represents the projection of a short fiber, and the fiber oritation can be determined by the angles $\theta$ and $\varphi$. Here, the parameter, $\varphi$, ranging from $0^{\circ}$ to $180^{\circ}$, is the angle between the projected fiber and the $x$-aixs in the $x-y$ plane. The parameter, $\theta$, ranging from $0^{\circ}$ to $90^{\circ}$, is the angle between the fiber and the $z$-aixs. According to the fiber angle in the threedimensional spherical coordinate system, the short fibers can be projected arbitrarily in the corresponding orthogonal plane coordinate system to establish a simplified model.

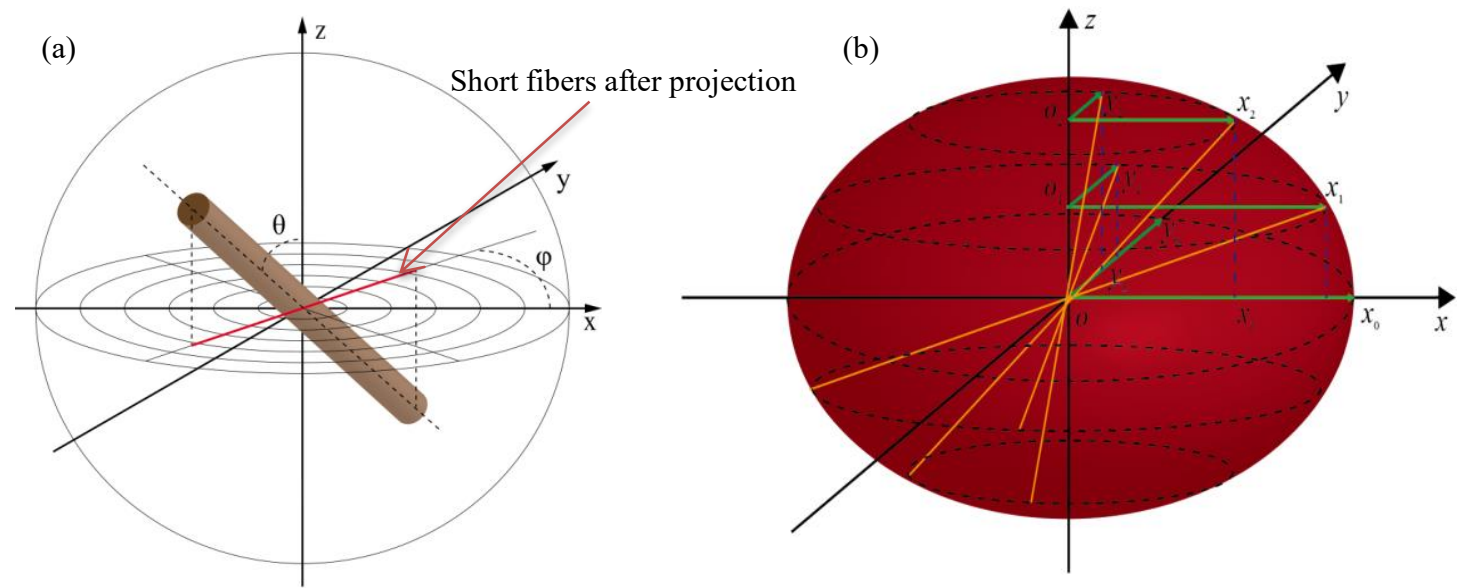

Fig. 6. Short fibers projection on the $x-y$ plane: (a) Schematic diagram of short fiber in CT test

(b) Schematic diagram of the simplified model

A mapping micro-topography of the SFRCs can be directly generated by employing the finite element model through the commercial micro-CT and image post-processing software AVIZO. However, the high demand of computational cost and poor efficiency had prevented researchers to follow this route due to the complex fiber arrangements. To simplify the microscopic model and improve the calculation efficiency, a simplified model with an ellipsoidal inclusion in the matrix materials is proposed to approximate the effective modulus and stress-strain relations, where fiber volume content and tri-axial ratio are the critical parameters. It should be noted that the research region with enough short fibers should be chosen in predicting effective properties of the SFRCs.

From the geometric structure of the ellipsoid inclusion, the intersections taken by planes that are perpendicular to a coordinate axis are ellipses. The ratio between the squared semi-major axis and the semi-minor axis of all the intersections is a constant. Therefore, the semi-axis dimensions of the ellipsoidal inclusion can be determined according to the projection of the randomly distributed short 
fibers on coordinate planes of the $x-y$ and the $y-z$, as shown in Fig. 6(b). For instance, fibers $o x_{2}$ and $o y_{2}$ are respectively projected on the $x-y$ plane, and their projection length on the $x-y$ plane can be easily acquired. After all the reinforcing short fibers are projected on the $x$-y and the $y-z$ planes, the semi-axis lengths along the coordinates can be determined by employing the follow equation.

$$
\bar{L}^{k}=\sum_{i=1}^{n} \sqrt{\frac{1}{n}\left(L_{i}^{k}\right)^{2}}(k=1,2,3)
$$

where $\bar{L}^{k}$ denotes the average length of the semi-axis along the three coordinates of $x$-, $y$ and $z$-directions. $L_{i}^{k}$ and $n$ represent the length and number of short fibers projected on the three coordinate planes, respectively.

Fig. 7 show the scatter plots and histograms, which are employed to present the orientation and length of the short fibers projected on the $x-y$ and $y-z$ planes. It should be pointed out that the angle here is associated to the fiber distributions, which is different from the cutting angle mentioned in Section 2.2. From Figs. 7(a)-(b), it can be found that the scatter plots projected on the $x-y$ and $y-z$ planes are obviously directional. In details, most of the fibers projected on the $x-y$ plane are approximate to $90^{\circ}(y$-axis) as shown in Fig. 7(a). While the fiber projected on the $y$ - $z$ plane as shown in Fig. 7(b) presents a more divergency. To further explain this phenomenon, the histograms as shown in Figs. 7(c)-(d) are used. It can be seen from Fig. 7(c) that the histogram presents a normal distribution that shows more short fibers in $90^{\circ}(y$-axis) than in any other fiber orientations. From the corresponding histogram as shown in Fig. 7 (d), it can be seen that a large number of fibers are orientated approximately in $90^{\circ}(z$-axis). By analyzing the data in the scatter plots within a reasonable range, as well as calculating the fiber length projected on the planes, a simplified ellipsoid model with a ratio of three axes 1:2:2.4 can be determined by employing Eq. (3).

(a)

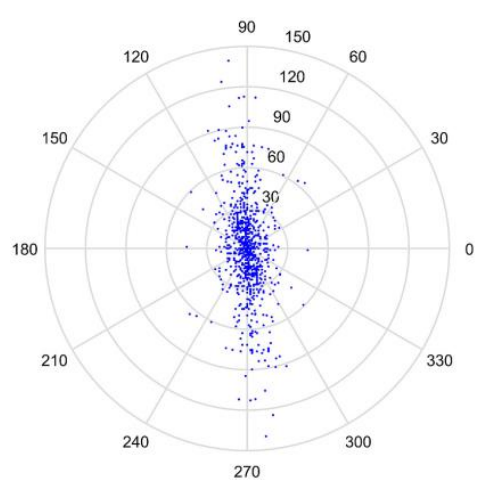

(c)

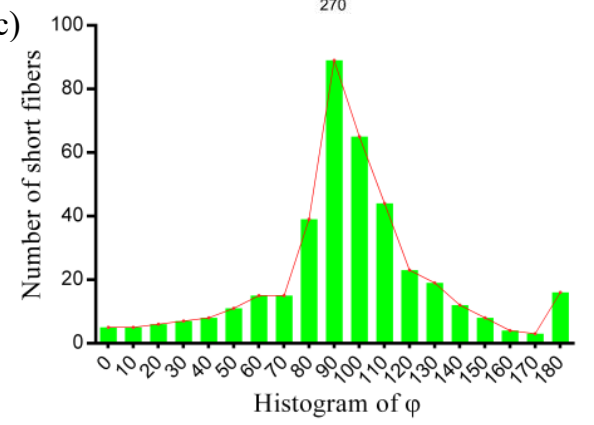

(b)

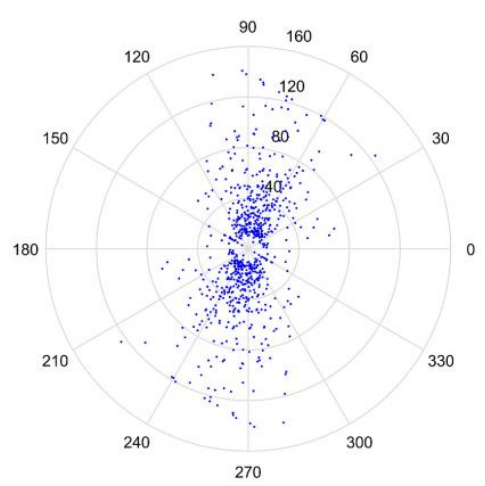

(d)

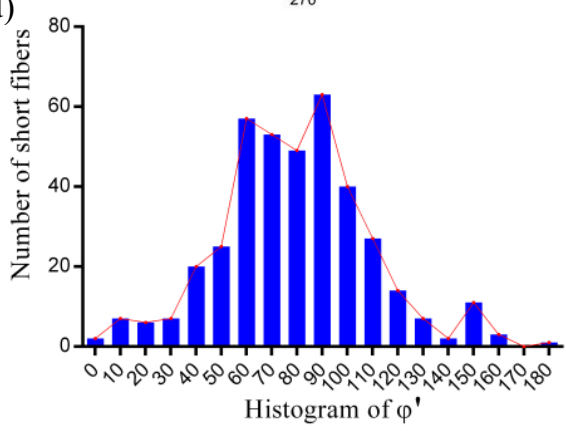

Fig. 7. Length and orientation for the projecting fibers: (a) Projecting fibers in the $x-y$ plane (b) Projecting fibers in the $y-z$ plane (c) The orientation distribution of short fibers projecting on the $x-y$ plane (d) The orientation distribution of short fibers projecting on the $y-z$ plane 
In order to deal with the uncoupled tension-shear in the Generalized Method of Cells, Bansal and Pindera et al. $[28,29]$ proposed a novel method to study the stress or strain distributions in the localized field by adding some higher order components. To overcome local stress concentration derived from quadrilateral or cubic elements, iso-parametric elements are introduced to discretize the representative volume element (RVE) [30-32]. At present, Ye, et al. [33] have extended the 2D parameterized FVDAM model to the 3D parameterized FVDAM model [34-35]. Herein, the threedimensional parametric FVDAM model is employed to analyze the mechanical behaviors of the SFRCs with a complex microscopic morphology as shown in Fig. 6(b). To fit the microscopic structure of the RVE, iso-parametric meshes are constructed by employing the linear interpolation function (see in Fig. 8). The range of the variables, $\eta, \xi$ and $\zeta$, in the parametric coordinate system is between -1 to 1 , can be written as follows:

$$
\begin{gathered}
y_{i}^{(q)}(\zeta, \eta, \xi)=\sum_{p=1}^{8} N_{p}(\zeta, \eta, \xi) y_{i}^{(p, q)}, i=1,2,3 \\
N_{1}(\zeta, \eta, \xi)=\frac{1}{8}(1-\zeta)(1-\eta)(1-\xi), N_{2}(\zeta, \eta, \xi)=\frac{1}{8}(1-\zeta)(1+\eta)(1-\xi), \\
N_{3}(\zeta, \eta, \xi)=\frac{1}{8}(1-\zeta)(1+\eta)(1+\xi), N_{4}(\zeta, \eta, \xi)=\frac{1}{8}(1-\zeta)(1-\eta)(1+\xi), \\
N_{5}(\zeta, \eta, \xi)=\frac{1}{8}(1+\zeta)(1-\eta)(1-\xi), N_{6}(\zeta, \eta, \xi)=\frac{1}{8}(1+\zeta)(1+\eta)(1-\xi), \\
N_{7}(\zeta, \eta, \xi)=\frac{1}{8}(1+\zeta)(1+\eta)(1+\xi), N_{8}(\zeta, \eta, \xi)=\frac{1}{8}(1+\zeta)(1-\eta)(1+\xi)
\end{gathered}
$$

According to the mapping relationship between the Cartesian and the parametric coordinate systems, as shown in Figs. 8(a)-(b), the Jacobian matrix is established as follows:

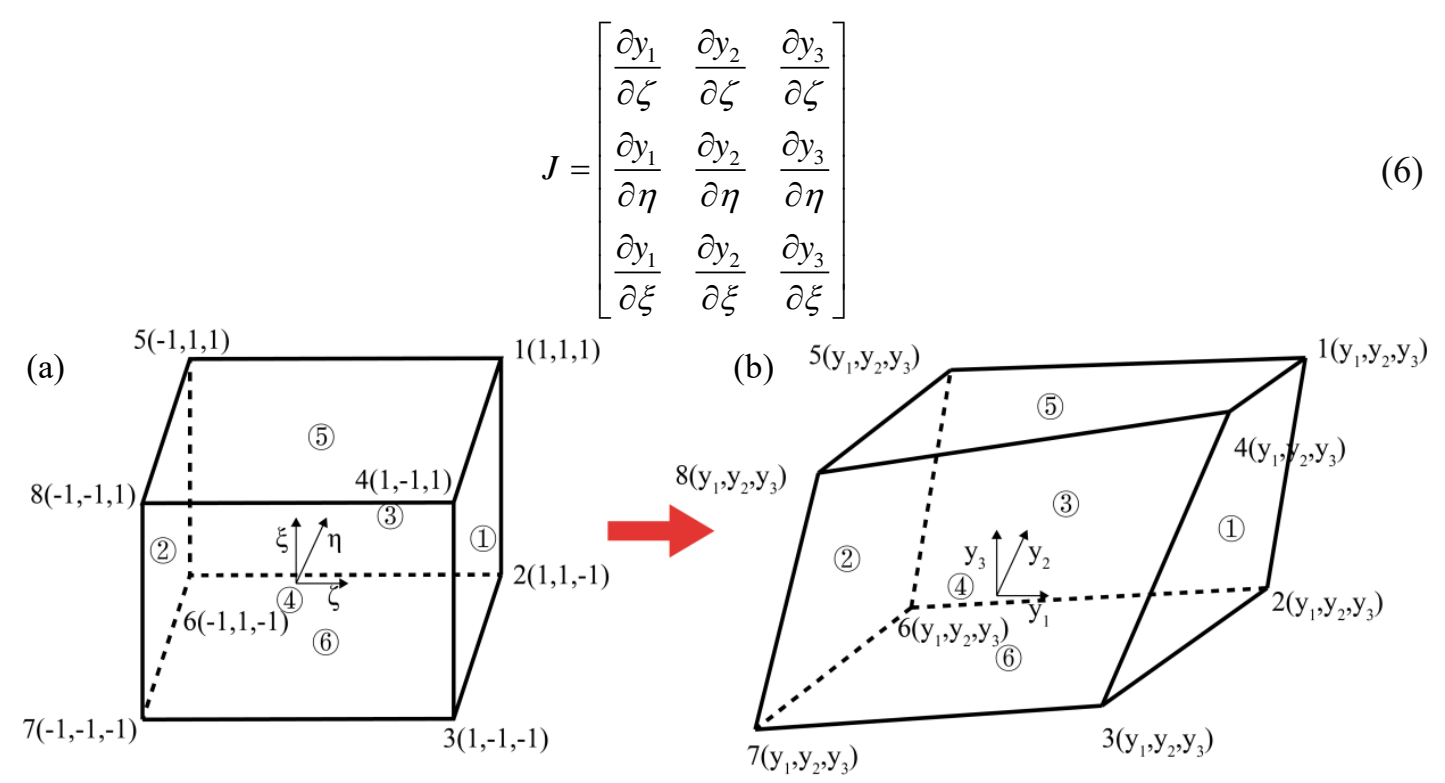

Fig. 8. Iso-parametric transformation: (a) The cubic mesh of the sub-cell (b) The isoparametric mesh of the sub-cell

where the parameter $p$ of the shape function $N$ represents the serial number of the corner points in the parametric element. The superscript $n$ represents the number of the sub-cells. Fig. 9(a) shows the discretized spherical inclusion with parametric meshes. For simplicity, 1/2 RVE of the composites is shown in Fig. 9(b). It should be pointed out that the RVE dimension will be determined once the microscopic structure of the composites is confirmed. 
(a)

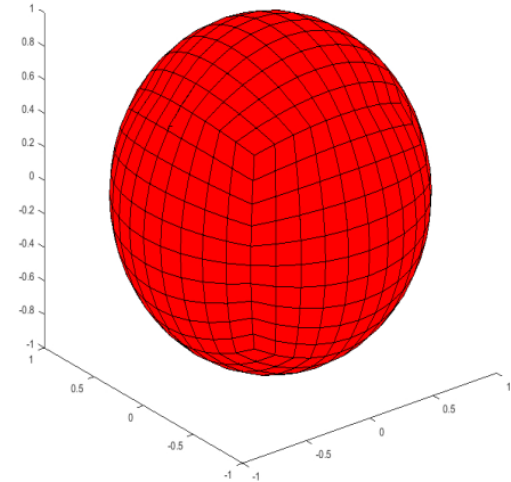

(b)

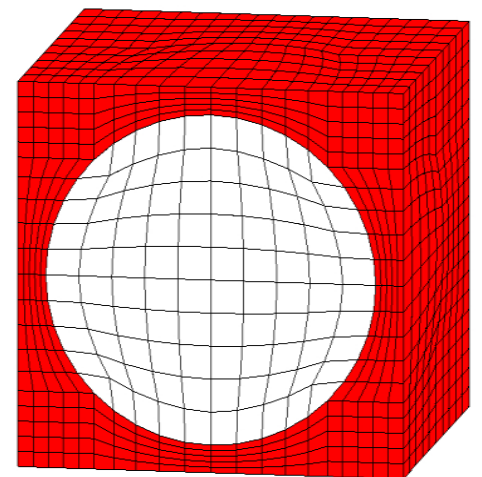

Fig. 9. Isoparametric RVE model: (a) Particle reinforced composites with parametric meshes

(b) 1/2 RVE of particle reinforced composites

The fluctuating displacement components of the discretized sub-cells can be further expressed in the parametric coordinate system with the Legendre polynomial in Eq. (7).

$$
\begin{aligned}
& u_{i}^{\prime(n)}(\zeta, \eta, \xi)=W_{i(000)}^{(n)}+\zeta W_{i(100)}^{(n)}+\eta W_{i(010)}^{(n)}+\xi W_{i(001)}^{(n)}+\frac{1}{2}\left(3 \zeta^{2}-1\right) W_{i(200)}^{(n)} \\
& +\frac{1}{2}\left(3 \eta^{2}-1\right) W_{i(020)}^{(n)}+\frac{1}{2}\left(3 \xi^{2}-1\right) W_{i(002)}^{(n)}
\end{aligned}
$$

where the subscript $i$ represents three local coordinate directions. The unknown micro-variables $W_{i(u v w)}^{(n)}$ are the intermediate variables of the sub-cell stresses and displacements. Here, the serial number in the parentheses of the micro-variables represents the high-order terms along different directions. To obtain the zero-order unknown micro-variables $W_{i(000)}^{(n)}$, the equilibrium equations should be satisfied in the $n$th sub-cell, as shown in Eq. (8).

$$
\int_{S} \mathbf{t}_{i}^{\mathrm{n}} \mathbf{d S}=\sum_{\mathrm{p}=1}^{6} \mathbf{S}_{\mathrm{p}} \mathbf{t}_{\mathrm{i}}^{\mathrm{p}}=\mathbf{0}
$$

Combined with the sub-cell surface-averaged stresses, surface displacements, as well as the unknown micro-variables, the displacement-stress relationship in the local coordinate system can be summarized as,

$$
\overline{\mathbf{t}}=\mathbf{N C} \overline{\boldsymbol{\varepsilon}}+\overline{\mathbf{A}} \mathbf{W}
$$

where

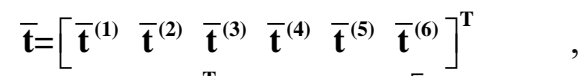$$
\mathbf{N}=\left[\begin{array}{llll}
\mathbf{n}^{(1)} & \mathbf{n}^{(2)} & \mathbf{n}^{(3)} & \mathbf{n}^{(4)} \mathbf{n}^{(5)} \mathbf{n}^{(6)}
\end{array}\right]^{\mathrm{T}}
$$

$\bar{\varepsilon}=\left[\begin{array}{llllll}\bar{\varepsilon}_{11} & \bar{\varepsilon}_{22} & \bar{\varepsilon}_{33} & \bar{\varepsilon}_{23} & \bar{\varepsilon}_{13} & \bar{\varepsilon}_{12}\end{array}\right]^{\mathrm{T}}$ and $\mathbf{W}_{\mathrm{i}}=\left[\begin{array}{llllll}\mathbf{W}_{\mathrm{i}(100)} & \mathbf{W}_{\mathrm{i}(010)} & \mathbf{W}_{\mathrm{i}(001)} & \mathbf{W}_{\mathrm{i}(200)} & \mathbf{W}_{\mathrm{i}(020)} & \mathbf{W}_{\mathrm{i}(002)}\end{array}\right]^{\mathrm{T}}$. Here, the matrix $\overline{\mathbf{t}}$ denotes the sub-cell stress and its superscripts represent six surfaces of the sub-cells. The matrix $\mathbf{N}$ includes the angles between sub-cell surface and global coordinate system. The matrices $\overline{\boldsymbol{\varepsilon}}$ and $\mathbf{W}$ are the macroscopic applied strain and the unknown micro-variables, respectively. The matrix $\overline{\mathbf{A}}$ consists of four different matrices, and the detailed expressions of them can be found in [33]. According to the procedure mentioned above, the local stress-displacement relationship is determined. In order to assemble the displacement-stress relationship of the sub-cells in the global stiffness matrix, the continuity and periodical conditions should be satisfied as follows:

$$
\mathbf{K} \overline{\mathbf{u}}=\Delta \mathbf{C} \overline{\boldsymbol{\varepsilon}}
$$

Combining the global stiffness matrix in Eq. (10) with the relationships between the unknown micro-variables and displacements, the sub-cell stresses and sub-cell displacements are calculated. Moreover, the volume-averaged stress-strain relations in each sub-cell element yield the following constitutive equations for the 3D composites, as shown in Eqs. (11)-(12). 


$$
\begin{gathered}
\overline{\boldsymbol{\sigma}}=\frac{1}{V} \int_{V} \boldsymbol{\sigma}(x) d V=\sum_{n=1}^{N} v^{n} \overline{\boldsymbol{\sigma}}^{(n)}=C^{*} \overline{\boldsymbol{\varepsilon}} \\
\mathbf{C}^{*}=\sum_{n=1}^{N} v^{n} \mathbf{C}^{\mathbf{n}} \mathbf{A}^{\mathbf{n}}
\end{gathered}
$$

where $\mathbf{C}^{*}$ and $\mathbf{C}^{n}$ donate the effective stiffness matrix and sub-cell stiffness in the local coordinate system, respectively. The symbol $v$ and $\mathbf{A}$ are the sub-cell volume and the Hill matrix. $\overline{\boldsymbol{\sigma}}$ and $\overline{\boldsymbol{\varepsilon}}$ are the effective stress and strain, respectively. Further details of the above parameters and matrices can be found in [34].

\subsection{Model validation}

\subsubsection{Ideal spherical model}

The post-processing procedure of the simplified model are performed on Matlab 2019(a) software. Moreover, the Intel Xeon ${ }^{\circledR}$ E5-2697A v4 2.6GHz Sixteen Core Processor with 64G memory workstation are employed to execute the numerical simulation. To improve the computational efficiency, the parallel computing is carried out on matrix formation and operation simultaneously. Glass/PEEK composites are considered and constituent material parameters are in Table. 1. According to the density of the constituent materials, volume fraction of the short fibers can be calculated. To discuss mesh density effects on the simulating results, $20 \times 20 \times 20$ and $38 \times 38 \times 38$ meshes were both considered to discretize the RVE. Numerical results indicate the relative error of elastic modulus between these two mesh densities is less than $1 \%$. Thus, to improve the calculation efficiency, $20 \times 20 \times 20$ meshes were selected for the following studies.

For a comparison, the modulus of the SFRCs $(6.40 \mathrm{GPa})$ provided by Ensinger Inc. is employed. The ideal spherical inclusion is assumed distributed in the matrix materials, and its stress and strain distributions are shown in Fig. 10. From the stress-strain relations in the RVE, the effective modulus of the simplified micromechanical model is estimated and 6.52GPa. Comparing with the modulus provided by the manufacturer, the relative error is less than 3\%. Therefore, it can be concluded that the simplified model can be used to effectively predict the mechanical properties of SFRCs.

(a)

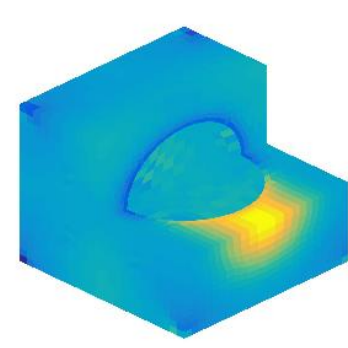

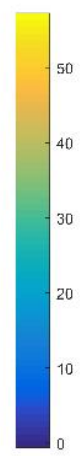

(b)

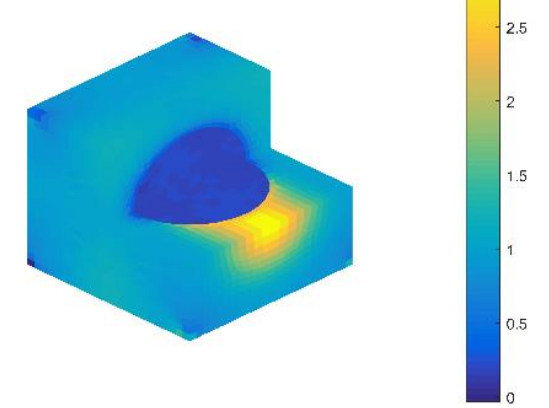

Fig. 10. Stress and strain distributions in composites with a spherical inclusion under the unidirectional strain of $1000 \mu \varepsilon$ : (a) Stress nephogram (MPa) (b) Strain nephogram

\subsubsection{Effectively simplified model}

During the material forming process, the resin flow leads to directionality of the short fibers. Naturally, the SFRCs present anisotropic properties. To describe this property, a simplified ellipsoid model is proposed here. According to the statistical results of the micro-CT in Section 3.2, the triaxial ratio of the simplified ellipsoid model is 1:2:2.4. Herein, the displacement loading is applied on the simplified RVE model, and the strain rate is $10^{-4} / \mathrm{s}$. Fig. 11(a) and Fig. 11(b) are the stress and strain distributions of the composites when the macroscopic strain reaches to $0.1 \%$, respectively. 
It should be noted that the cutting angles, i.e., $0^{\circ}$ and $90^{\circ}$ in Section 2.2 refer, respectively, to the major and minor axis directions in the simplified ellipsoid model. To calculate the elastic modulus of the $45^{\circ}$ specimens, it is necessary to rotate the equivalent stiffness matrix of the simplified model with respect to the middle-axis as follows [29],

$$
\left[T_{i j}\right]^{-1}=\left[\begin{array}{cccccc}
\cos ^{2} \theta & \sin ^{2} \theta & 0 & 0 & 0 & -\sin 2 \theta \\
\sin ^{2} \theta & \cos ^{2} \theta & 0 & 0 & 0 & \sin 2 \theta \\
0 & 0 & 1 & 0 & 0 & 0 \\
0 & 0 & 0 & \cos \theta & \sin \theta & 0 \\
0 & 0 & 0 & -\sin \theta & \cos \theta & 0 \\
\frac{\sin 2 \theta}{2} & -\frac{\sin 2 \theta}{2} & 0 & 0 & 0 & \cos 2 \theta
\end{array}\right]
$$

(a)

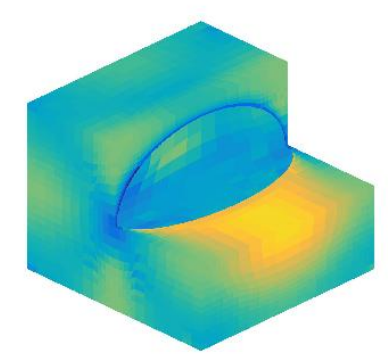

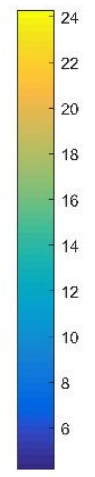

(b)

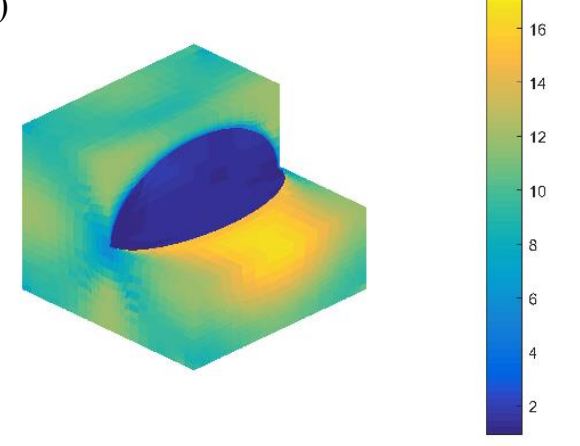

Fig. 11. Stress and strain distributions in composites with an ellipsoidal inclusion under the unidirectional strain of $1000 \mu \varepsilon$ : (a) Stress nephogram (MPa) (b) Strain nephogram

Through the calculations by the 3D parameterized FVDAM, the elastic moduli along the major axis $\left(0^{\circ}\right.$ samples) and minor axis $\left(90^{\circ}\right.$ samples) are $7.634 \mathrm{GPa}$ and $5.517 \mathrm{GPa}$, respectively. The numerical result of the elastic modulus along $45^{\circ}$ direction is $6.207 \mathrm{GPa}$ by employing Eqs. (13)(14). For comparisons, both the experimental and the numerical results are shown in Fig. 12. It should be point out that the degree of experimental samples in Fig. 12 is defined as the cutting angle, as shown in Fig. 2. Numerical results of the elastic moduli are higher than the experimental ones. To explain this difference, the two-dimensional slice from the micro-CT is shown in Fig. 13, where, the blue and red phases are voids and short fibers, respectively. It can be seen that there are a large number of internal voids in composites. In other words, this difference is derived from neglecting the internal voids in the microscopic model. However, it should be pointed out that the difference between the numerical results and the experimental data tends to be consistent in all the three directions. In other words, the internal voids result in a consistent decrease of effective moduli for the SFRCs in different directions. In details, compared with the effective moduli of the theoretical model, the voids result in nearly $25 \%$ reduction in the three directions. 


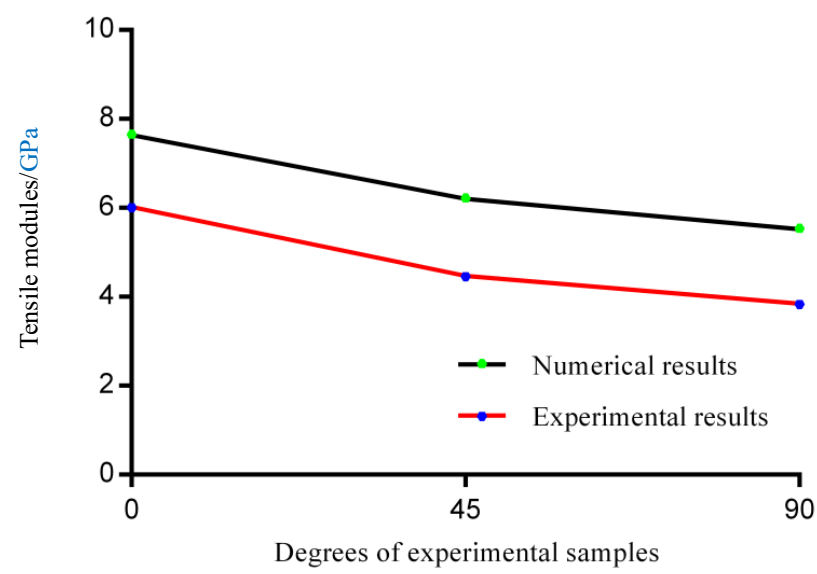

Fig. 12. The comparison of elastic modulus in different orientations for SFRCs between numerical results and experimental results

(a)

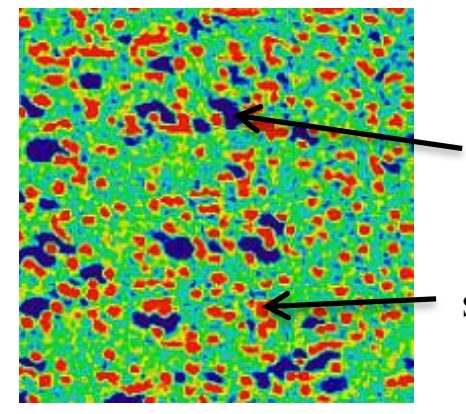

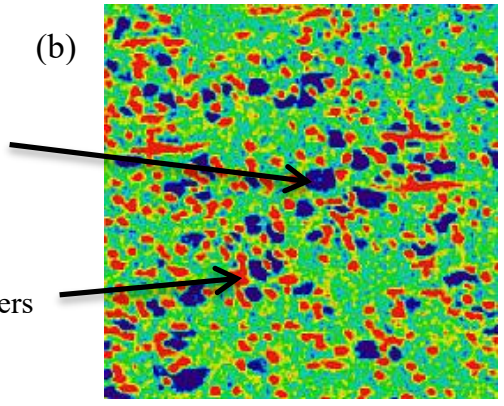

(b)

ef micro-CT scanning

\section{SEM tests}

\subsection{Sample preparations}

The Scanning electron microscope JSM-6390A shown in Fig. 14(a) is employed to capture the failure modes and micro-morphology of the SFCRs. Due to the height limitation, all the test samples are truncated perpendicular to the fracture plane as shown in Fig. 14(b). To easier discern the microscopic damages, the objective samples are treated by spraying gold to increase their conductivity. Here, the acceleration voltage of the scanning electron microscope is kept as a constant at $15 \mathrm{kV}$ in scanning the damaged surface of the samples. Since the fiber diameter is about $14 \mu \mathrm{m}$, the magnification of electron microscopic observation keeps between 200 and 800 times to clearly capture their microscopic morphologies.

(a)

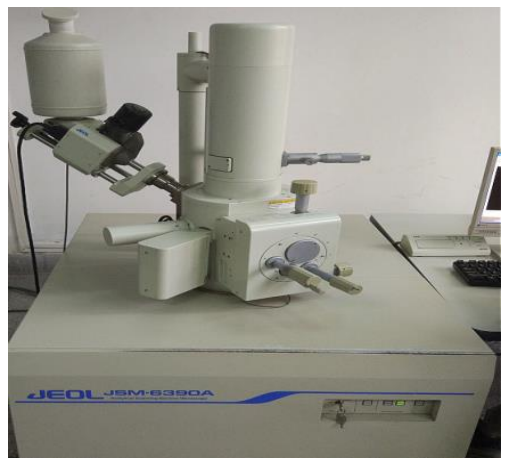

(b)

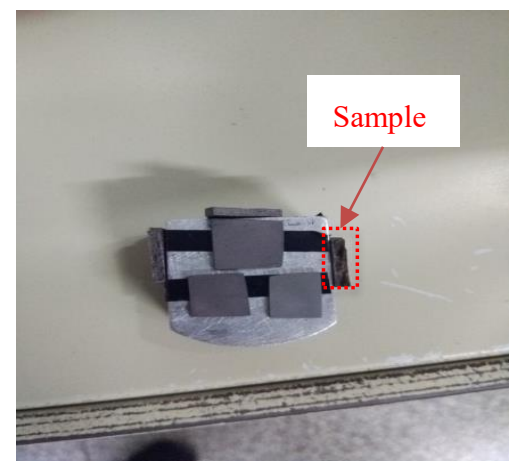

Fig. 14. Experimental equipment and samples: (a) JSM-6390A scanning electronic microscope (b) SEM samples on the objective table 


\subsection{Experimental analysis}

Fig. 15(a) shows one of the test samples, the damaged section of which is not rough with many localized deep dents. Moreover, three-dimensional cracks have appeared on the fracture surface at the initial stage of the two-dimensional longitudinal tensile experiment. It can be concluded that the cracks are closely associated with the micro-morphology and the short fiber distributions. In addition, the short glass fibers, which parallel to the macroscopic loading direction, show obvious brittle fracture as shown in Fig. 15(b). Fig. 15(c) is a zoomed-in picture to show fiber fractures in Fig. 15(b). It can be seen that the short fibers, which are not parallel to the loading direction, show ductile fractures in terms of the unevenly fractured cross-sections. Similar definition refers to the ductile fracture has been mentioned by Fu et al. [36]. Based on the analyses mentioned above, it can be said that short-fiber orientation plays an important role in the process of microscopic damage. Moreover, the failures of the SFRCs are closely related to the ductile property of the matrix, as well as the short fibers that are not parallel to the loading direction. Correspondingly, the fibers parallel to the load direction have a great influence on the brittle failure behaviors of the SFRCs. In general, the coupled brittle-ductile failure of the SFRCs depends closely on the random distributions of the short fibers in the three-dimensional space.

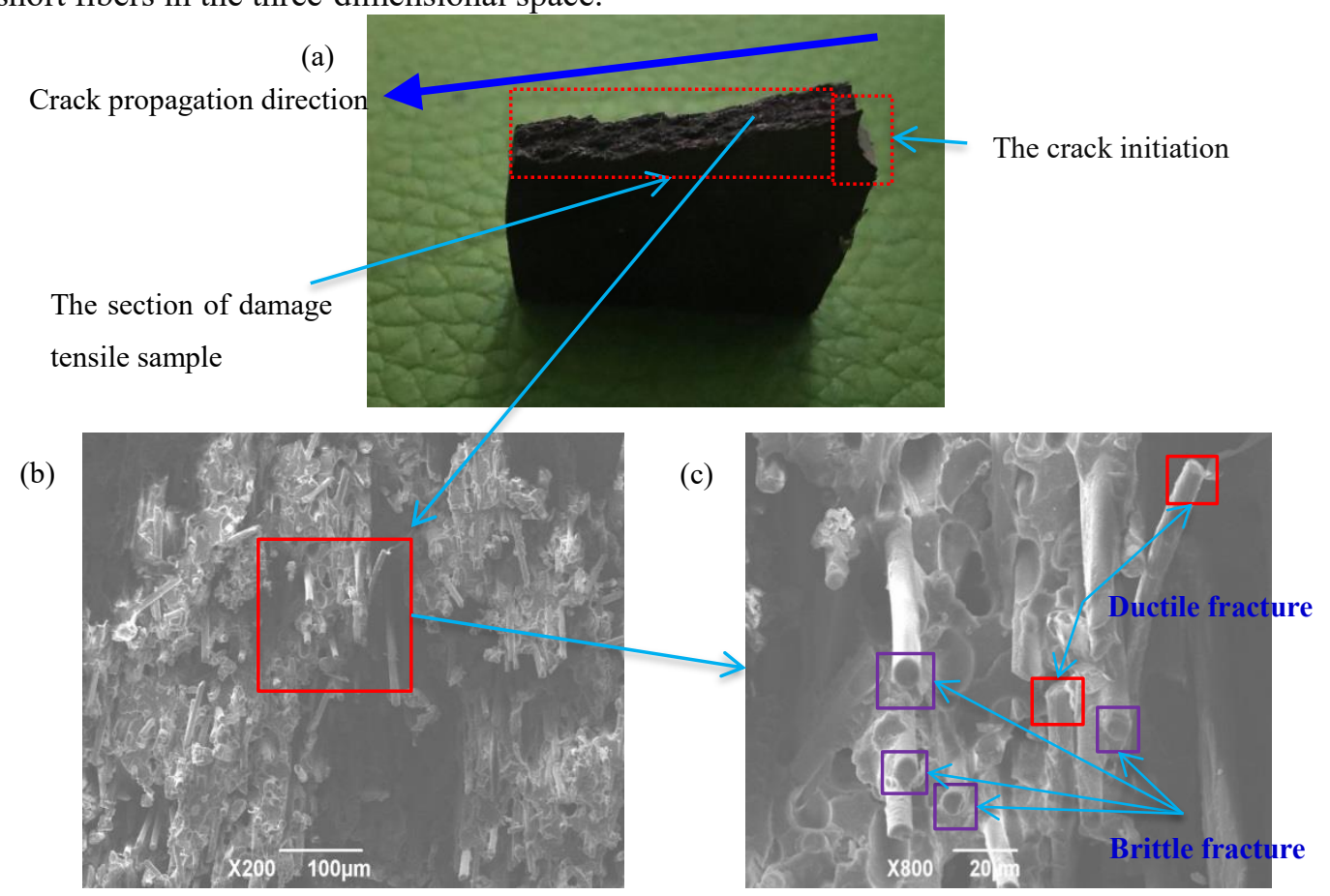

Fig. 15. Macroscopic and microscopic damage morphology of samples: (a) SEM samples with the fracture cross-section (b) Micro-morphology of fracture section (c) Partial enlarged details

\section{Conclusions}

In this paper, a simplified microscopic model is proposed, and the failure mechanisms of the SFRCs are studied. The anisotropic mechanical behaviors of the SFRCs are investigated first by quasi-static tensile tests, and the micro-morphology of short fibers randomly distributed in the polymer matrix is acquired by using micro-CT. The anisotropic mechanical behaviors caused by the spatial distributions of short fibers are then investigated by the proposed simplified model. Finally, the effect of spatial random distribution of short fibers on the failure of composites is discussed according to the experimental results of the SEM. The conclusions are summarized as follows: 
1) An effective modeling scheme is proposed to effectively predict elastic modulus of composites with randomly distributed short fibers. In studying elastic modulus of anisotropic composites, a nearly $25 \%$ relative error between the proposed model and experimental data is found due to the ignorance of initial voids in the composites during the theoretical modeling.

2) Spatially random distribution short fibers lead to anisotropic behaviors of SFRCs made by CM technology. Material forming process has a great influence on the defect formation of SFRCs.

3) Random arrangement of short fibers results in a remarkable improvement of ductile property of the SFRCs.

Constrained by the cost and time required from the experiments, the authors only tested samples of $0^{\circ}, 45^{\circ}$ and $90^{\circ}$ to validate the proposed model. The differences between the numerical results and the experimental data are mainly due to the ignorance of initial voids in the composites. Future research is required to include this effect as well as nonlinear behaviors of the composites in other directions.

\section{Acknowledgments}

This work was supported by the National Natural Science Foundation of China (No. 51675397). The National Natural Science Foundation of Shaanxi Province (No. 2018JZ5005). China Scholarship Council (No. 201706965037). Fundamental Research Funds for the Central Universities (No. JB180414), The 111 Project (No. B14042). DY would like to thank the financial support from EPSRC (EP/P017169/1) for the Zeiss X-ray micro-CT equipment and Avizo software.

Conflicts of Interest: The authors declare no competing interests.

Data Availability Statement: The raw/processed data required to reproduce these findings cannot be shared at this time as the data also forms part of an ongoing study.

\section{References}

[1] Shi PP. Singular integral equation method for 2D fracture analysis of orthotropic solids containing doubly periodic strip-like cracks on rectangular lattice arrays under longitudinal shear loading. Appl Math Model. 2020; 77: 1460-1473.

[2] Yang ZB, Radzienski M, Kudela P, Ostachowicz W. Damage detection in beam-like composite structures via Chebyshev pseudo spectral modal curvature. Compos Struct 2017; 168: 1-12.

[3] Ye J, Chu C, Cai H, Hou X, Shi B, Tian S, et al. A multi-scale model for studying failure mechanisms of composite wind turbine blades. Compos Struct 2019;212:220-9.

[4] Ye J.J., Wang Y.W., Li Z.W., Saafi M., Jia F., Huang B., Ye J.Q. Failure analysis of fiber-reinforced composites subjected to coupled thermo-mechanical loading. Compos Struct 2020;235:111756..

[5] Raney JR, Compton BG, Mueller J, Ober TJ, Shea K, Lewis JA. Rotational 3D printing of damage-tolerant composites with programmable mechanics. Proc Natl Acad Sci U S A 2018; 115: 1198-203.

[6] Ferreira RTL, Amatte IC, Dutra TA, Bürger D. Experimental characterization and micrography of 3D printed PLA and PLA reinforced with short carbon fibers. Compos Part B Eng 2017; 124: 88-100.

[7] Hambach M, Volkmer D. Properties of 3D-printed fiber-reinforced Portland cement paste. Cem Concr Compos 2017; 79: 62-70.

[8] Trofimov A, Mishurova T, Lanzoni L, Radi E, Bruno G, Sevostianov I. Microstructural analysis and mechanical properties of concrete reinforced with polymer short fibers. Int J Eng Sci 2018; 133: 210-8.

[9] Afroughsabet V, Biolzi L, Ozbakkaloglu T. High-performance fiber-reinforced concrete: a review. vol. 51. Springer US; 2016.

[10] Wu Z, Shi C, He W, Wang D. Static and dynamic compressive properties of ultra-high performance concrete (UHPC) with hybrid steel fiber reinforcements. Cem Concr Compos 2017; 79: 148-57. 
[11] Arif MF, Meraghni F, Chemisky Y, Despringre N, Robert G. In situ damage mechanisms investigation of PA66/GF30 composite: Effect of relative humidity. Compos Part B Eng 2014; 58:487-95.

[12] Rolland H, Saintier N, Raphael I, Lenoir N, King A, Robert G. Fatigue damage mechanisms of short fiber reinforced PA66 as observed by in-situ synchrotron X-ray microtomography. Compos Part B Eng 2018; 143: 217-29.

[13] Shi Y, Tushtev K, Koch D. Characterization of mechanical properties under shear load of a short-carbon-fiberreinforced C/SiC ceramic. J Ceram Sci Technol 2015; 6:183-9.

[14] Hu X, Fang J, Xu F, Dong B, Xiao Y, Wang L. Real internal microstructure based key mechanism analysis on the micro-damage process of short fibre-reinforced composites. Sci Rep 2016; 6: 1-9.

[15] Lee DJ, Oh H, Song YS, Youn JR. Analysis of effective elastic modulus for multiphased hybrid composites. Compos Sci Technol 2012; 72: 278-83.

[16] Shen H, Nutt S, Hull D. Direct observation and measurement of fiber architecture in short fiber-polymer composite foam through micro-CT imaging. Compos Sci Technol 2004; 64: 2113-20.

[17] Wu K, Wan L, Zhang H, Yang D. Numerical simulation of the injection molding process of short fiber composites by an integrated particle approach. Int J Adv Manuf Technol 2018; 97: 3479-91.

[18] Nguyen Thi TB, Morioka M, Yokoyama A, Hamanaka S, Yamashita K, Nonomura C. Measurement of fiber orientation distribution in injection-molded short-glass-fiber composites using X-ray computed tomography. $\mathrm{J}$ Mater Process Technol 2015; 219:1-9.

[19] Yu X, Gu B, Zhang B. Effects of short fiber tip geometry and inhomogeneous interphase on the stress distribution of rubber matrix sealing composites. J Appl Polym Sci 2015; 132: 1-8.

[20] Kompiš V, Murčinková Z. Thermal properties of short fibre composites modeled by meshless method. Adv Mater Sci Eng 2014; 2014.

[21] Ma H, Xia LW, Qin QH. Computational model for short-fiber composites with eigenstrain formulation of boundary integral equations. Appl Math Mech (English Ed) 2008; 29: 757-67.

[22] Lei HF, Zhang ZQ, Liu B. Effect of fiber arrangement on mechanical properties of short fiber reinforced composites. Compos Sci Technol 2012; 72: 506-14.

[23] Dean A, Sahraee S, Reinoso J, Rolfes R. A new invariant-based thermo-plastic model for finite deformation analysis of short fibre reinforced composites: Development and numerical aspects. Compos Part B Eng 2017; 125: 241-58.

[24] Dean A, Reinoso J, Sahraee S, Rolfes R. An invariant-based anisotropic material model for short fiberreinforced thermoplastics: Coupled thermo-plastic formulation. Compos Part A Appl Sci Manuf 2016; 90: 186-99.

[25] Tanaka K, Oharada K, Yamada D, Shimizu K. Fatigue crack propagation in short-carbon-fiber reinforced plastics evaluated based on anisotropic fracture mechanics. Int J Fatigue 2016; 92: 415-25.

[26] Müller V, Brylka B, Dillenberger F, Glöckner R, Kolling S, Böhlke T. Homogenization of elastic properties of short-fiber reinforced composites based on measured microstructure data. J Compos Mater 2016; 50: 297 312.

[27] Qin QH, Swain MV. A micro-mechanics model of dentin mechanical properties. Biomaterials 2004;25:508190.

[28] Bansal Y, Pindera MJ. A second look at the higher-order theory for periodic multiphase materials. J Appl Mech Trans ASME 2005; 72: 177-95.

[29] Bansal Y, Pindera MJ. Finite-volume direct averaging micromechanics of heterogeneous materials with elastic-plastic phases. Int J Plast 2006; 22: 775-825.

[30] Ye J, Cai H, Wang Y, Jing Z, Shi B, Qiu Y, et al. Effective mechanical properties of piezoelectric- 
piezomagnetic hybrid smart composites. J Intell Mater Syst Struct 2018; 29: 1711-23.

[31] Chen Q, Chen X, Zhai Z, Yang Z. A new and general formulation of three-dimensional finite-volume micromechanics for particulate reinforced composites with viscoplastic phases. Compos Part B Eng 2016; 85: 216-32.

[32] Cai H, Ye J, Wang Y, Jia F, Hong Y, Tian S, et al. Matrix failures effect on damage evolution of particle reinforced composites. Mech Adv Mater Struct 2019: 1-13.

[33] Yang DH, Yang ZB, Zhai Z, Chen XF. Homogenization and localization of ratcheting behavior of composite materials and structures with the thermal residual stress effect. Mater 2019; 12(3048):1-20.

[34] Ye J, Hong Y, Cai H, Wang Y, Zhai Z, Shi B. A new three-dimensional parametric FVDAM for investigating the effective elastic moduli of particle-reinforced composites with interphase. Mech Adv Mater Struct 2019; 26: $1870-80$.

[35] Chen Q, Wang G, Chen X. Three-Dimensional Parametric Finite-Volume Homogenization of Periodic Materials with Multi-Scale Structural Applications. Int J Appl Mech 2018; 10.

[36] Fu SY, Mai YW, Lauke B, Yue CY. Synergistic effect on the fracture toughness of hybrid short glass fiber and short carbon fiber reinforced polypropylene composites. Mater Sci Eng A 2002; 323(1-2):326-335. 